\title{
GEOVISUALIZAÇÃO DE MAPAS HISTÓRICOS
}

\author{
José Flávio Morais Castro
}

Resumo: A geovisualização de mapas históricos envolve um conjunto de técnicas cartográficas e computacionais para representar a informação espacial pretérita. Este trabalho tem por objetivo aplicar uma técnica alternativa de geovisualização de mapa histórico em ambientes WEBGIS e Google Earth. Pretende-se também apresentar de forma sucinta, resultados parciais da metodologia de georreferenciamento de mapas históricos que vem sendo desenvolvida na Linha de Pesquisa em Cartografia Histórica e Geoprocessamento do Programa de PósGraduação em Geografia - Tratamento da Informação Espacial da PUC Minas nos últimos anos. Esta pesquisa aplica métodos da Comunicação Cartográfica (Semiologia Gráfica) e da Visualização Cartográfica no processo de Geovisualização de mapas históricos, especialmente mapas do Brasil dos séculos XVIII e XIX, em ambientes WEBGIS e Google Earth. Assim, após o georreferenciamento do mapa histórico em ARC $\mathrm{GIS}^{\circledR}$, o tratamento foi realizado por meio da superposição em imagens de satélites georreferenciadas, como o Google Earth Pro.

Palavra-chave: Geovisualização; cartografia histórica; mapa histórico; semiologia gráfica: geoprocessamento.

\section{GEOVISUALIZATION OF HISTORICAL MAPS}

\begin{abstract}
The geovisualization of historical maps involves a set of cartographic and computational techniques to represent the past spatial information. This work aims to apply an alternative historical map geovisualization technique in WEBGIS and Google Earth environments. It is also intended to present, in a succinct way, partial results of the work that has been developed in the Line of Research in Historical Cartography and Geoprocessing of the Postgraduate Program in Geography Treatment of Spatial Information of PUC Minas in recent years. This research applies methods of Cartographic Communication (Graphic Semiology) and Cartographic Visualization in the process of Geovisualization of historical maps, especially Brazilian maps of the XVIII and XIX centuries, in WEBGIS and Google Earth environments. Thus, after the georeferencing of the historical map in ARC GIS ${ }$, the treatment was performed by superposition in geo-referenced satellite images, such as Google Earth Pro.
\end{abstract}

Keywords: Geovisualization; historical cartography; historic map; graphic semiology: geoprocessing.

\footnotetext{
${ }^{1}$ Professor Adjunto IV do Programa de Pós-graduação em Geografia - Tratamento da Informação Espacial da PUC Minas (joseflavio@pucminas.br)
} 


\section{INTRODUÇÃO}

A geovisualização de mapas históricos envolve um conjunto de técnicas cartográficas e computacionais para representar a informação espacial pretérita.

A partir dos anos 1960, a Cartografia passou a adotar no ensino e na pesquisa, entre outros métodos, a Semiologia Gráfica (BERTIN, 1967) no tratamento da informação espacial. Concebido como um dos métodos de alfabetização cartográfica e como meio de comunicação, os mapas produzidos nesta cartografia atuam cognitivamente como elementos estratégicos e como importantes instrumentos de pesquisa, que permitem identificar padrões e dinâmicas espaciais.

Com a introdução dos recursos computacionais na Cartografia, por volta dos anos de 1970, o processo de análise da informação tornou-se interativo, principalmente com o uso dos Sistemas de Informações Geográficas (SIG's) e da internet.

Este trabalho tem por objetivo aplicar uma técnica alternativa de geovisualização de mapa histórico em ambientes WEBGIS e Google Earth. Pretende-se também apresentar de forma sucinta, resultados parciais da metodologia de georreferenciamento de mapas históricos que vem sendo desenvolvida na Linha de Pesquisa em Cartografia Histórica e Geoprocessamento do Programa de Pós-Graduação em Geografia - Tratamento da Informação Espacial da PUC Minas nos últimos anos.

As possíveis relações entre Cartografia Histórica e Geoprocessamento surgiram em 1996, a partir da publicação do Mapa da Capitania de Minas Gerais com a divisa de suas comarcas, de 1778 (ROCHA, 1995) - (Figura 1). Concomitantemente, surgiu a pergunta fundamental: como georreferenciá-lo em um Sistema de Informações Geográficas (SIG)?

Figura 1 - Mapa da Comarca de Villa Rica de 1778

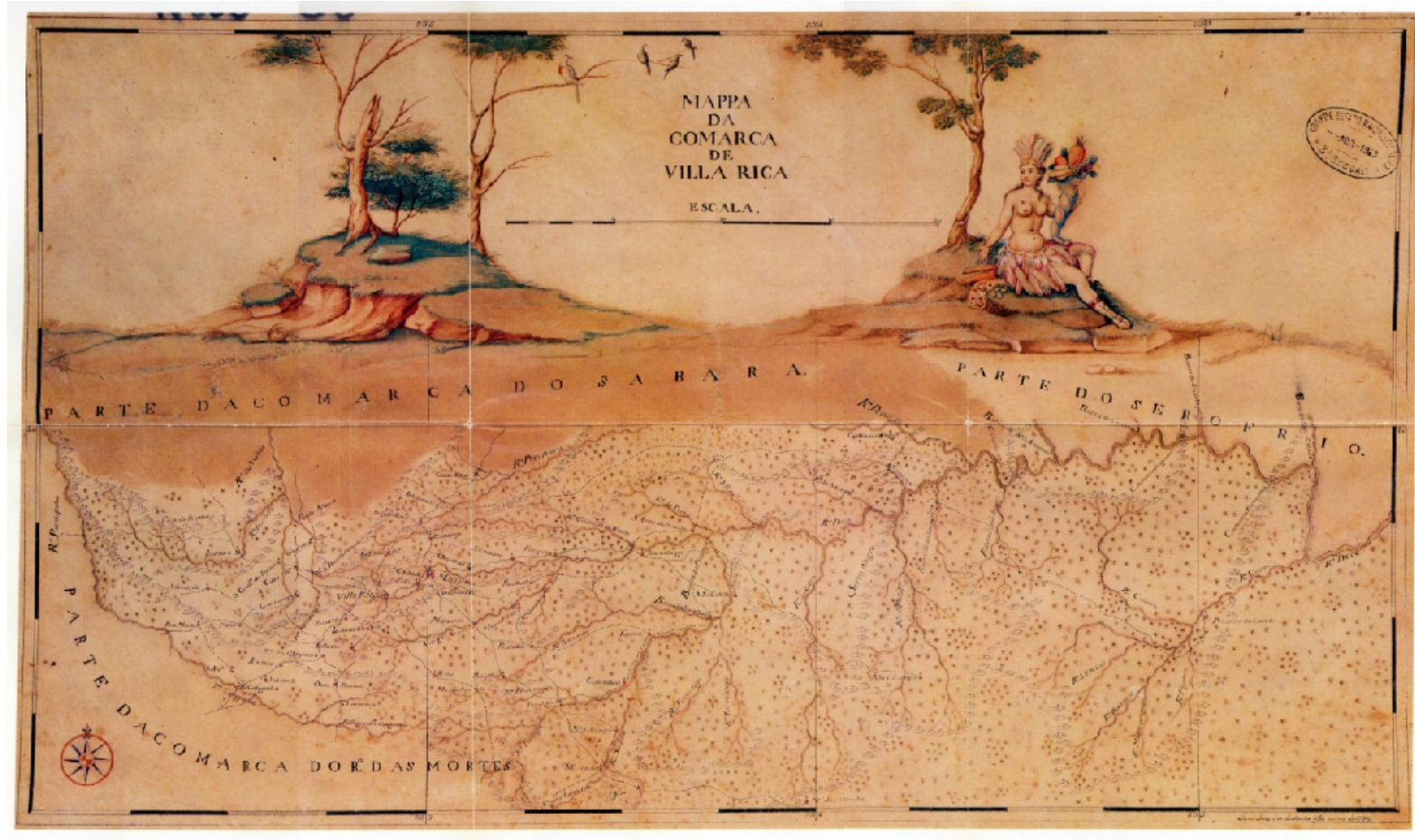

Fonte: Rocha (1995). 
Uma iniciativa ocorreu em 1997 a partir da vetorização do mapa da Comarca de Villa Rica, de 1778, em software de desenho do tipo desktop mapping (Figura 2), ou seja, ausência de georreferenciamento.

Figura 2 - Vetorização do mapa da Comarca de Villa Rica, de 1778

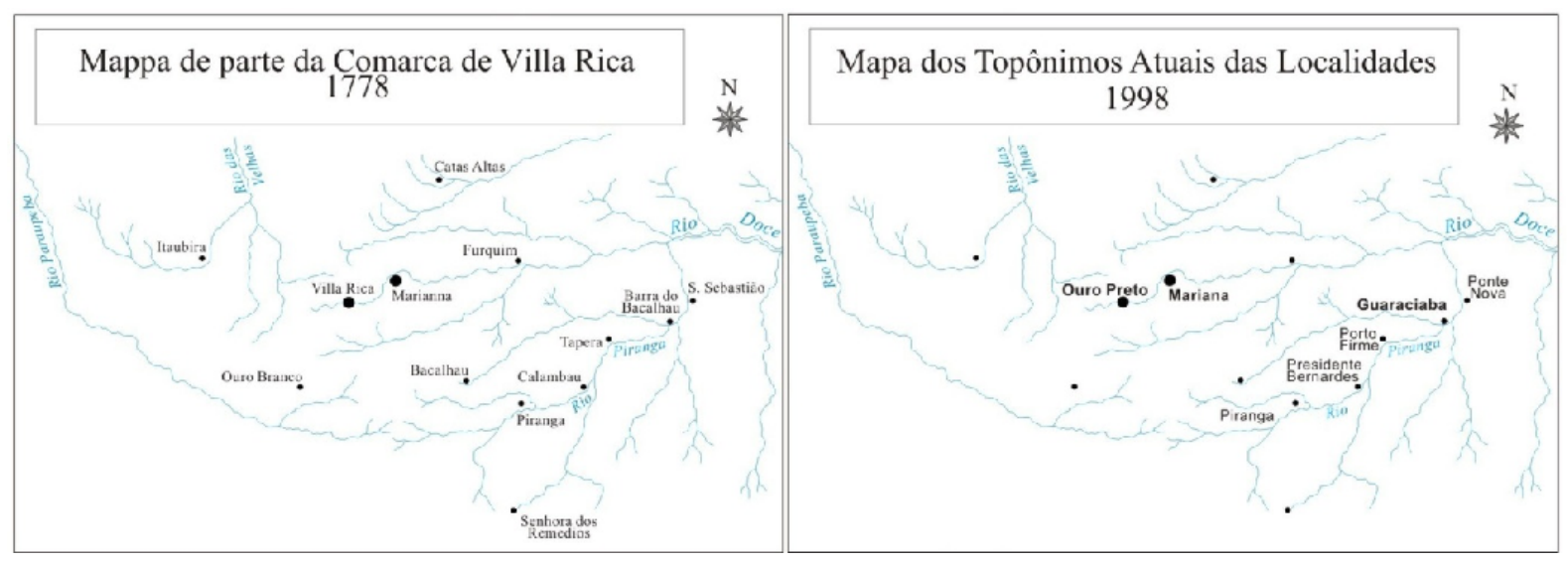

Fonte: Castro (1998).

Em 2006, após sucessivas experiências, parte da solução foi encontrada, culminando com a criação de uma metodologia de georreferenciamento e de tratamento de mapas históricos em SIG (Figura 3), e com o desenvolvimento da linha de pesquisa intitulada Cartografia Histórica e Geoprocessamento no Programa de Pós-Graduação em Geografia - Tratamento da Informação Espacial da PUC Minas.

Figura 3 - Metodologia de georreferenciamento de mapas históricos

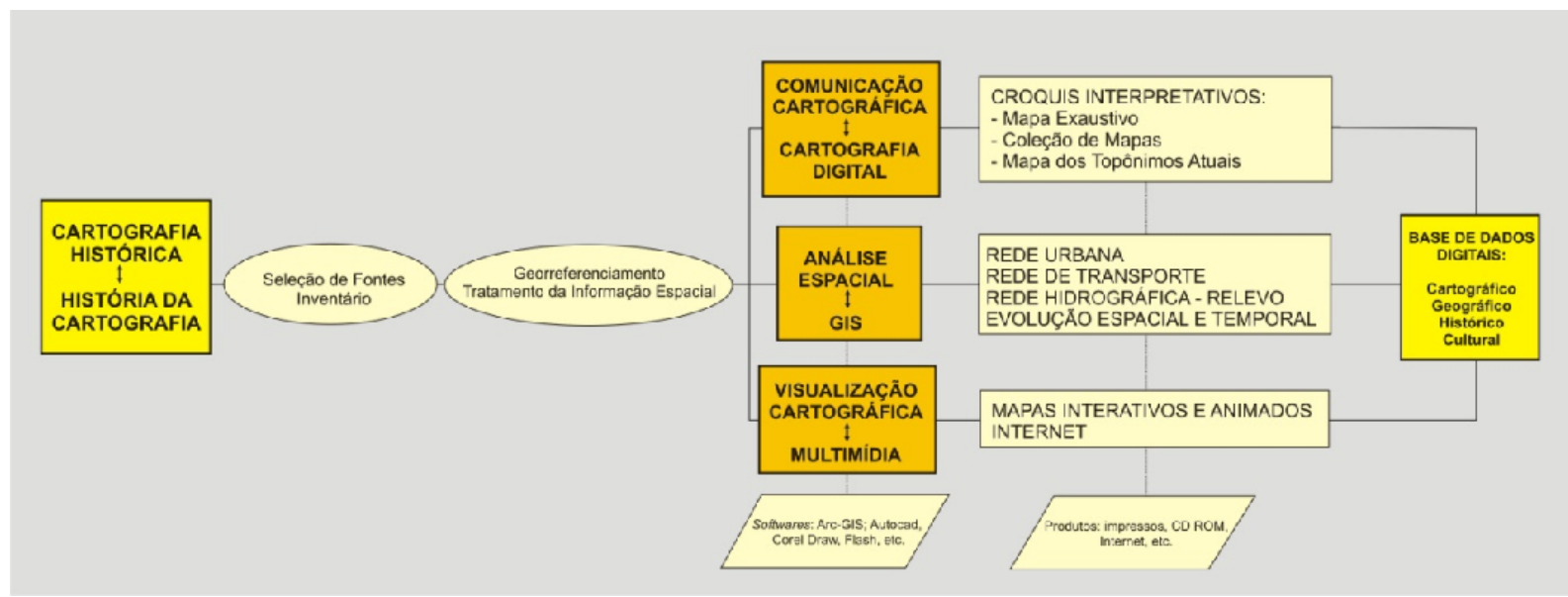

Fonte: Castro et al. (2006).

Nos últimos anos, foram realizadas variadas pesquisas decorrentes de projetos isolados ou na forma de orientações de alunos de iniciação científica, mestrado e doutorado, que culminaram com apresentações de comunicações em eventos nacionais e internacionais, e publicações em periódicos e anais. 


\section{METODOLOGIA}

A proposta de georreferenciamento de mapas históricos foi modificada em 2011 e apresenta três etapas: inventário das fontes, processo e produto (Figura 4).

Figura 4 - Metodologia de análise de mapas históricos por técnicas de geoprocessamento

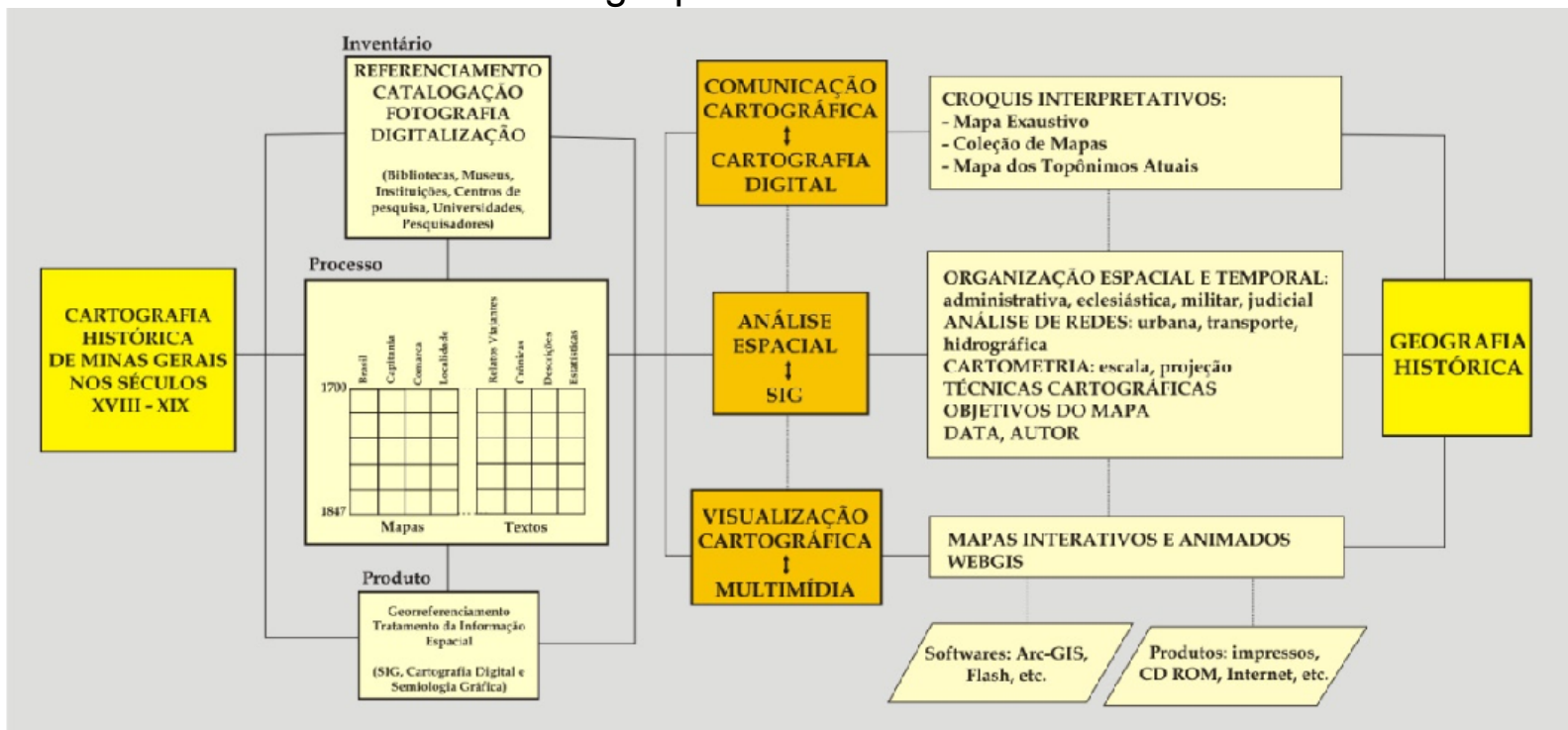

Fonte: modificada de Castro (2011).

O inventário da Cartografia histórica de Minas Gerais, conforme o roteiro metodológico, inicia-se pela consulta de arquivos e acervos de documentos históricos. Nesses levantamentos, tendo por base os aspectos geográficos e históricos, o foco deve ser direcionado, em um primeiro momento, para a identificação, descrição, catalogação, digitalização e criação do banco de dados dos mapas e textos.

O processo envolve a análise dos documentos, entre 1700 e 1822, com atenção especial às autorias, datações, escalas de representação (localidade, comarca, capitania e Brasil) e às técnicas cartográficas empregadas no mapeamento.

Paralelamente, visando à contextualização histórica e geográfica da Capitania de Minas Gerais, são identificados trabalhos que fundamentam a pesquisa, por exemplo: Lima Júnior (1965), Rocha (1995), Antonil (2001), Costa et al. (2002), Cortesão (2009), entre outros.

Tomando-se por base o geoprocessamento e a Semiologia gráfica, a fase de produto envolve o georreferenciamento e o tratamento da informação espacial em três etapas: comunicação cartográfica e cartografia digital; análise espacial e SIG; e visualização cartográfica e WEBGIS, ou Geovisualização.

A primeira etapa envolve a utilização da comunicação cartográfica e dos recursos da semiologia gráfica por meio digital, criando-se banco de dados georreferenciados e elaborando-se croquis interpretativos: mapa exaustivo e coleção de mapas (BERTIN, 1988, p. 47). 
A segunda etapa, a partir do banco de dados digital produzido, realiza-se a análise espacial, tais como: organização espacial e temporal, cartogramas, hierarquia urbana, cartometria, redes, fluxos, entre outras aplicações em Sistemas de Informações Geográficas (SIGs)

A terceira etapa, envolve a publicação da informação produzida por meio da visualização cartográfica e da WEBGIS em Google Earth. Assim, após o georreferenciamento do mapa histórico em um SIG, o tratamento pode ser realizado por meio da superposição em imagens de satélites georreferenciadas, como o Google Earth Pro.

\section{GEOVISUALIZAÇÃO}

Dentre as variadas concepções adotadas na cartografia convencional destaca-se o Sistema de Comunicação Cartográfica (OLIVEIRA, 1978; SIMIELLI, 1986; KOLACNY, 1994; BOARD, 1994; KOEMAN, 1995; PETCHENIK, 1995) que consiste na representação do mundo real por meio de mapas, com ênfase nas concepções do cartógrafo e do usuário de mapas.

Uma das linguagens da comunicação cartográfica, a Semiologia (do grego semeion = sinal, signo, símbolo), estuda os sistemas de sinais que o homem utiliza no seio da vida social: línguas, códigos, sinalizações, entre outros (BERTIN, 1967). Como parte integrante destes sistemas de sinais, a Representação Gráfica é a parte da Semiologia que tem por objetivo transcrever uma informação por meio de um sistema de símbolos, que exercem dupla função: atuam concomitantemente como memória artificial e como instrumento de pesquisa.

A partir da década de 1970, grande parte dos conceitos e estruturas da cartografia convencional foram transformados para o formato digital, tornando o processo de análise da informação espacial dinâmico e interativo, por meio do uso de métodos e técnicas da Cartografia Digital, dos Sistemas de Informações Geográficas (SIG's) e da Visualização Cartográfica ou Geovisualização (Figura 5). 
Figura 5 - Interfaces entre conceitos e estruturas da cartografia convencional e da cartografia digital, fundamentais em SIG

\begin{tabular}{|c|c|c|c|}
\hline $\begin{array}{l}\text { INFORMAÇÃO } \\
\text { (palavra-chave) }\end{array}$ & $\begin{array}{l}\text { CARTOGRAFIA } \\
\text { CONVENCIONAL }\end{array}$ & $\begin{array}{l}\text { CARTOGRAFIA } \\
\text { DIGITAL }\end{array}$ & GIS \\
\hline $\begin{array}{c}\text { MODOS } \\
\text { DE } \\
\text { IMPLANTAÇÃO }\end{array}$ & $\begin{array}{l}\text { Ponto } \\
\text { Linha } \\
\text { Área }\end{array}$ & $\begin{array}{l}\text { Ponto } \\
\text { Linha (arco) } \\
\text { Poligono }\end{array}$ & \multirow{5}{*}{$\begin{array}{c}\text { METODOLOGIA } \\
\text { CLASSIFICAÇÃO } \\
\text { REGIONALIZAÇÃOO } \\
\text { PADRÔES } \\
\text { ANÁLISE ESTATISTICA } \\
\text { Univariada e Multivariada }\end{array}$} \\
\hline ESCALA & $\begin{array}{l}\text { Detalhe/Generalização } \\
\text { Nominal, Ordinal, Intervalo, Razão }\end{array}$ & Resolução Espacial & \\
\hline PROJEÇÃO & $\begin{array}{l}\text { Coordenadas Esféricas } \\
\text { Coordenadas Planas }\end{array}$ & Georreferenciamento & \\
\hline $\begin{array}{l}\text { DISTRIBUIÇÃO } \\
\text { ESPACIAL }\end{array}$ & $\begin{array}{l}\text { Discreta (Cartograma Coroplético) } \\
\text { Contínua (Cartograma Isoplético) }\end{array}$ & $\begin{array}{l}\text { Raster ou matricial (pixel) } \\
\text { Vetor (ponto, linha, área) }\end{array}$ & \\
\hline MATRIZ GEOGRÁFICA & Tabela de Dados & Banco de Dados (DBMS) & \\
\hline $\begin{array}{c}\text { REPRESENTAÇÃO } \\
\text { DE } \\
\text { VOLUME }\end{array}$ & $\begin{array}{l}\text { Bloco Diagrama } \\
\text { Maquete }\end{array}$ & Modelo 3D (DTM) & \multirow{3}{*}{$\begin{array}{c}\text { Operadores Lógicos } \\
\text { da Álgebra Booleana } \\
\text { (and, or, not) } \\
\text { Lógica Fuzzy } \\
\text { Redes Neurais } \\
\text { Artificiais }\end{array}$} \\
\hline CAMADA, PLANO & Mesa de Luz & LAYER & \\
\hline TEORIA & $\begin{array}{l}\text { COMUNICAÇÃO } \\
\text { CARTOGRÁFICA }\end{array}$ & $\begin{array}{l}\text { VISUALIZAÇÃO } \\
\text { CARTOGRÁFICA }\end{array}$ & \\
\hline
\end{tabular}

Fonte: elaborada pelo autor em 2000.

A Visualização Cartográfica surgiu no final da década de 1980, em decorrência dos avanços das técnicas computacionais, tornando-se uma alternativa de exploração dinâmica e interativa dos bancos de dados digitais.

Visualização é um termo com muitos significados. De maneira geral "to make visible" pode ser considerada, entre outras concepções possíveis, como uma categoria que pertence à cartografia. O termo visualização científica foi adotado com o significado estrito de tecnologia computadorizada avançada para facilitar o ato de "tornar visíveis" dados científicos e conceitos (MACEACHREN, 1995).

Segundo Wood e Brodlie (1994) apud Schmidt et al. (2007), o termo visualização científica computacional apareceu pela primeira vez em 1987 na publicação da National Science Foundation, em um relatório intitulado Visualization in Scientific Computing. Em seguida, estudos sobre técnicas de visualização e aplicação de texturas às imagens computacionais passaram a constituir um segmento de pesquisa chamado Visualization in Scientific Computing, ou simplesmente ViSC.

MacEachren (op. cit.) desenvolveu um modelo tridimensional de interação espacial homem-mapa, que define a aplicação ideal para visualização e comunicação. As dimensões das interações espaciais são definidas por uma tríade contínua: o uso do mapa privado (feito sob medida, elaborado para um indivíduo), para o público (designado para um público amplo), o uso do mapa direcionado para revelações desconhecidas (exploração) versus mostrar o conhecido (apresentação) e o uso de mapas que têm alta interação versus baixa interação (Figura 6). Não existe um limite claro nesta interação homem-mapa. Toda visualização com mapa envolve alguma comunicação e toda comunicação com mapa envolve alguma visualização. A distinção está na ênfase. 
Figura 6 - Cartografia: representada por um espaço cúbico no uso do mapa, no qual a visualização e a comunicação ocupam polos opostos

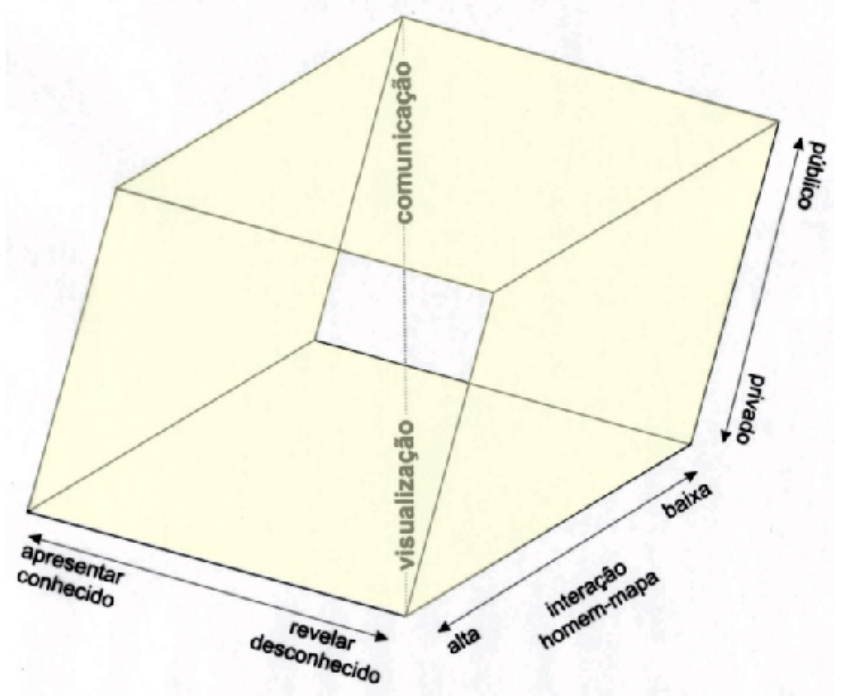

Fonte: MacEachren (1995).

A visualização consiste na criação de imagens gráficas por computador, que permitem análises multidimensionais. Tem sido amplamente considerada como um método por computador que incorpora coleta de dados, organização, modelagem e representação. A visualização é baseada na habilidade humana para impor ordem e identificar padrões.

Como consequência da análise estatística, a visualização é usada por uma variedade de disciplinas, especialmente a Cartografia, e está fortemente influenciada pelas variadas formas de análise de dados e de interfaces como a interatividade e a animação. A visualização cartográfica, às vezes chamada de visualização geográfica, é interpretada como sendo o uso de técnicas similares para exibição de mapas (PETERSON, 1995).

Interatividade e animação são palavras-chave no processo de visualização cartográfica. Segundo Peterson (1995), o mapa interativo é uma forma de apresentação do mapa digital que permite visualizar lugares e distribuições e estabelecer interfaces do uso intuitivo, fundamentado em símbolos gráficos, e um dispositivo para exibição de mapas simultaneamente que incluem comandos para produzir zoom e explorar diferentes áreas, com vídeo-clips, imagem e som de lugares. Para o referido autor, a animação é uma arte gráfica que ocorre no tempo e a manifestação da dinâmica visual que envolve diretamente a exibição, movimento ou troca.

Usando técnicas de visualização cartográfica, o usuário pode determinar os seguintes tipos de informação (SCHMIDT et al. 2007):

- Onde uma feição específica ou atributo está localizada;

- O que está na localização específica;

- Como feições em um ou mais layers estão relacionadas;

- Se existem anomalias nos dados,e;

- Se existem vários momentos diferentes, é possível avaliar as mudanças através do tempo. 
Para este conjunto de técnicas cartográficas e computacionais tem sido atribuído na literatura especializada o termo Geovisualização (GVis).

O termo "Geovisualização" surgiu pela primeira vez em uma comissão da ICA (International Cartographic Association) e foi emprgado por MacEachren e Kraak (2001), que pode ser descrito como um domínio limitado que envolve a análise visual, de síntese e de representação de dados geoespaciais, por meio da integração da cartografia com outras áreas de representação e análise, incluindo visualização científica, análise de imagens, análise exploratória de dados e GIScience (DYKES et al., 2005).

De forma abrangente, "a geovisualização implica em múltiplas representações de grandes e complexos conjunto de dados em tempo real" (LONGLEY et al. 2013, p.324).

Segundo Castro (2011), com o desenvolvimento do Google Earth por volta de 2005, um importante passo foi dado na aceitação do público em geral, em relação ao manuseio de informações geográficas, o que gerou um ganho até mesmo na padronização dos dados geoespaciais para a Web. A partir disso, o padrão KML (Key-Hole Markup Language) foi criado, inicialmente para ser a base de dados do Google Earth, mas devido a sua rápida aceitação ele está atualmente sendo mantido pelo OGC. O sucesso do KML se explica em parte por ser baseado no padrão XML, que é um padrão já consolidado para troca de informações na Web de acordo com Mathiak e Kupfer (2004). Em linhas gerais, a WEBGIS consiste em um conjunto de sistemas para representação e disponibilização cartográfica de informações espaciais, por meio de interfaces com a internet.

\section{GEOVISUALIZAÇÃO DE MAPAS HISTÓRICOS}

Para a aplicação da proposta metodológica de georreferenciamento de mapas históricos são utilizados, como exemplo, os mapas de Parte da Capitania de Minas Gerais, elaborados pelos padres matemáticos Domingos Capassi e Diogo Soares em 1734/35 (COSTA, 2004).

Os padres matemáticos elaboraram quatro mapas da região mineradora referenciados ao meridiano do Rio de Janeiro. A articulação dos quatro mapas representa, ao sul, a região de Ouro Preto e os rios Paraopeba e Velhas, e ao norte, a região diamantina e os rios Jequitinhonha e Araçuaí; portanto, o mapeamento recobre, pioneiramente, a região mineradora com sua organização urbana, as redes hidrográfica e viária (Figura 7).

Na primeira etapa, os mapas dos padres matemáticos, articulados com base no meridiano do Rio de Janeiro, são georreferenciados e vetorizados no ARC GIS ${ }^{\circledR}$, datum SAD 69 (South America Datum 1969), convertendo-se o meridiano do Rio de Janeiro para o meridiano de Greenwich (Figura 8).

Neste processo, são gerados layers (camadas) temáticos e vetorizados os elementos: rios, estradas, vilas, paróquias, capelas, bem como, o relevo e a vegetação, adequando-se a simbologia à original.

Os recursos do geoprocessamento e da cartografia temática, especialmente a Semiologia Gráfica (BERTIN, 1967; BERTIN 1988), permitem a superposição dos fenômenos em mapa único (mapa exaustivo) e a elaboração de um mapa para cada fenômeno mapeado no mapa exaustivo (coleção de mapas). Trata-se de um 
processo de desconstrução do espaço cartografado, minimizando-se os desafios de identificação e tratamento da informação mapeada (Figura 9).

A análise dos mapas, elaborados em escala regional, revela a organização espacial de parte da Capitania de Minas Gerais, bem como o processo de uso e ocupação do solo no início do século XVIII, fortemente influenciado pela extração do ouro; permite a identificação das relações entre os assentamentos urbanos, especialmente as Capelas, e a rede viária, ambos diretamente associados ao curso dos principais rios: das Velhas, Jequitinhonha e Araçuaí, caracterizando a região mineradora; permite ainda a identificação da distribuição espacial das Vilas e a concentração de Paróquias em torno de Ouro Preto, caracterizando o centro do poder administrativo e eclesiástico da capitania (CASTRO, 2014).

$\mathrm{Na}$ segunda etapa, são aplicadas técnicas cartométricas nos mapas dos padres matemáticos (Figura 10). A exatidão dos mapas históricos (GASPAR, 2000, 2009) pode ser avaliada nas pesquisas em Cartografia, por meio do uso de técnicas cartométricas.

A Cartometria é o campo da Cartografia que trata das medições e cálculo de valores numéricos relativos aos mapas e cartas, tais como: distâncias, áreas, direções, entre outras operações (GASPAR, 2009, p. 9).

Dentre os softwares disponíveis, o MapAnalyst ${ }^{\circledR}$ (JENNY; HURNI, 2011) ${ }^{2}$ vem sendo utilizado na avaliação da precisão do mapa histórico em relação ao atual, por meio de técnicas de interpolação ligadas aos vetores de deslocamentos e à distorção da grade de coordenadas.

Utilizando uma amostra de 23 pontos de controle nos mapas antigo e atual (Figura 10), relacionada às principais vilas e paróquias, bem como as confluências de alguns rios, a rede geográfica de meridianos e paralelos implícita foi interpolada. Os pontos de controle foram associados aos topônimos antigos e atuais. O mapa representam as distorções da grade de coordenadas e os descolamentos de vetores ocorridos no mapeamento da capitania em 1734, principalmente na longitude, desvios inerentes às dificuldades técnicas de levantamento no século XVIII. $\quad \mathrm{Na}$ associação de coordenadas antigas e atuais do mapa, note-se que os paralelos e os meridianos são curvas, com ângulos variáveis e que houve um ligeiro desvio para oeste.

$\mathrm{Na}$ terceira etapa, uma possibilidade de aplicação de geovisualização consiste em salvar arquivos, raster ou vetoriais, em formato KML e georreferencialos em Google Earth Pro (Figura 11). Esta técnica inovadora de apresentação de resultados de pesquisa por meio da internet, permite inúmeras manipulações e associações de informações pelo usuário.

Assim, o passo seguinte da pesquisa focará na interatividade com o usuário, oferecendo alternativas de determinação de variados tipos de informações georreferenciadas, especialmente para professores, criando-se metodologias de ensino de cartografia em Geografia e História por meio da WEB.

\footnotetext{
${ }^{2}$ Disponível para download em: http://www.ika.ethz.ch/mapanalyst/index.html.
} 
Geovisualização de mapas...

Figura 7 - Articulação dos mapas dos padres matemáticos - 1734/35

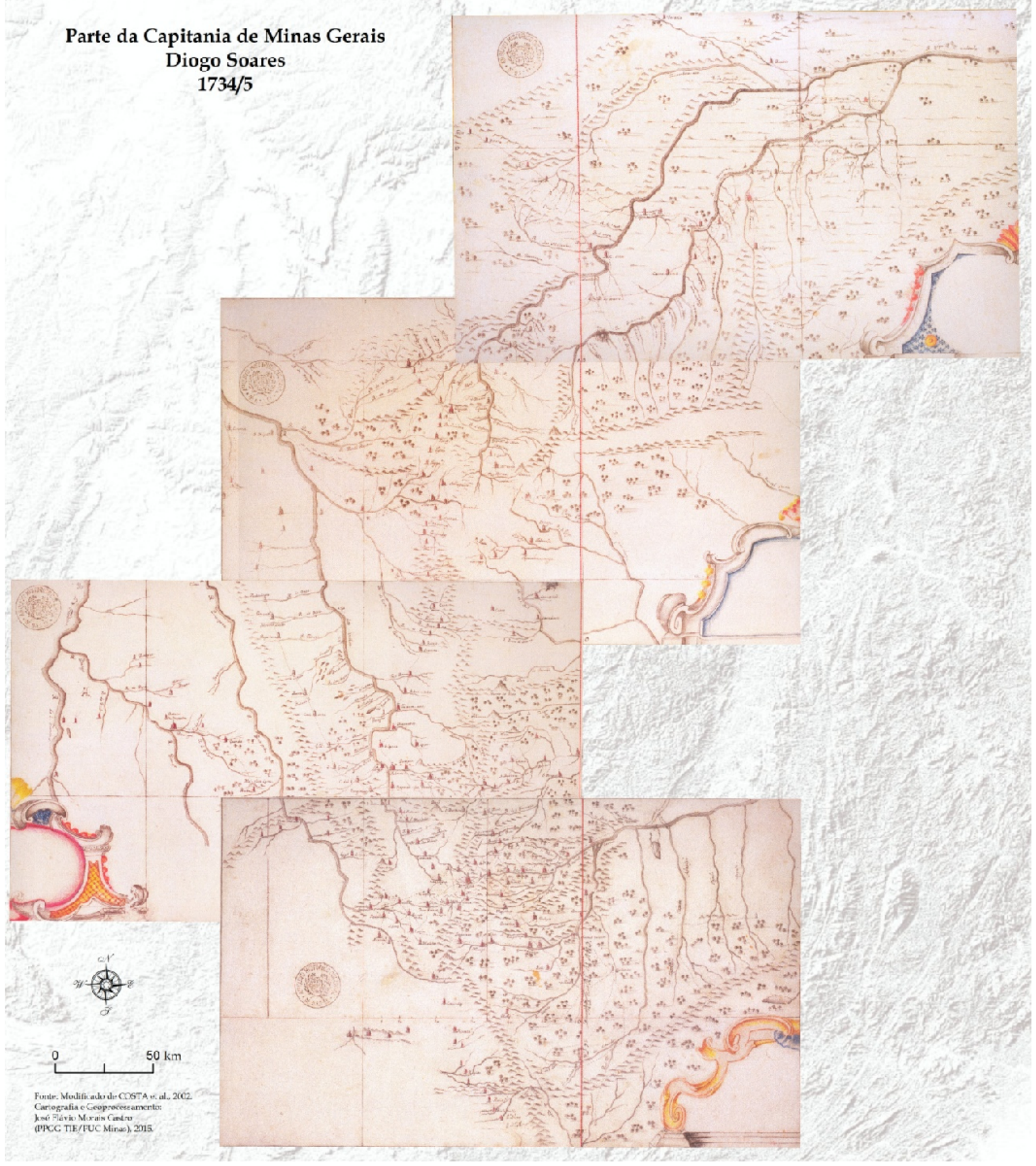

Fonte: Modificado de Costa (2004). 
Figura 8 - Cálculo para transformação de projeção dos mapas padres matemáticos - 1734/35, referenciados ao meridiano do Rio de Janeiro para o meridiano de Greenwich

Meridiano de Greenwich

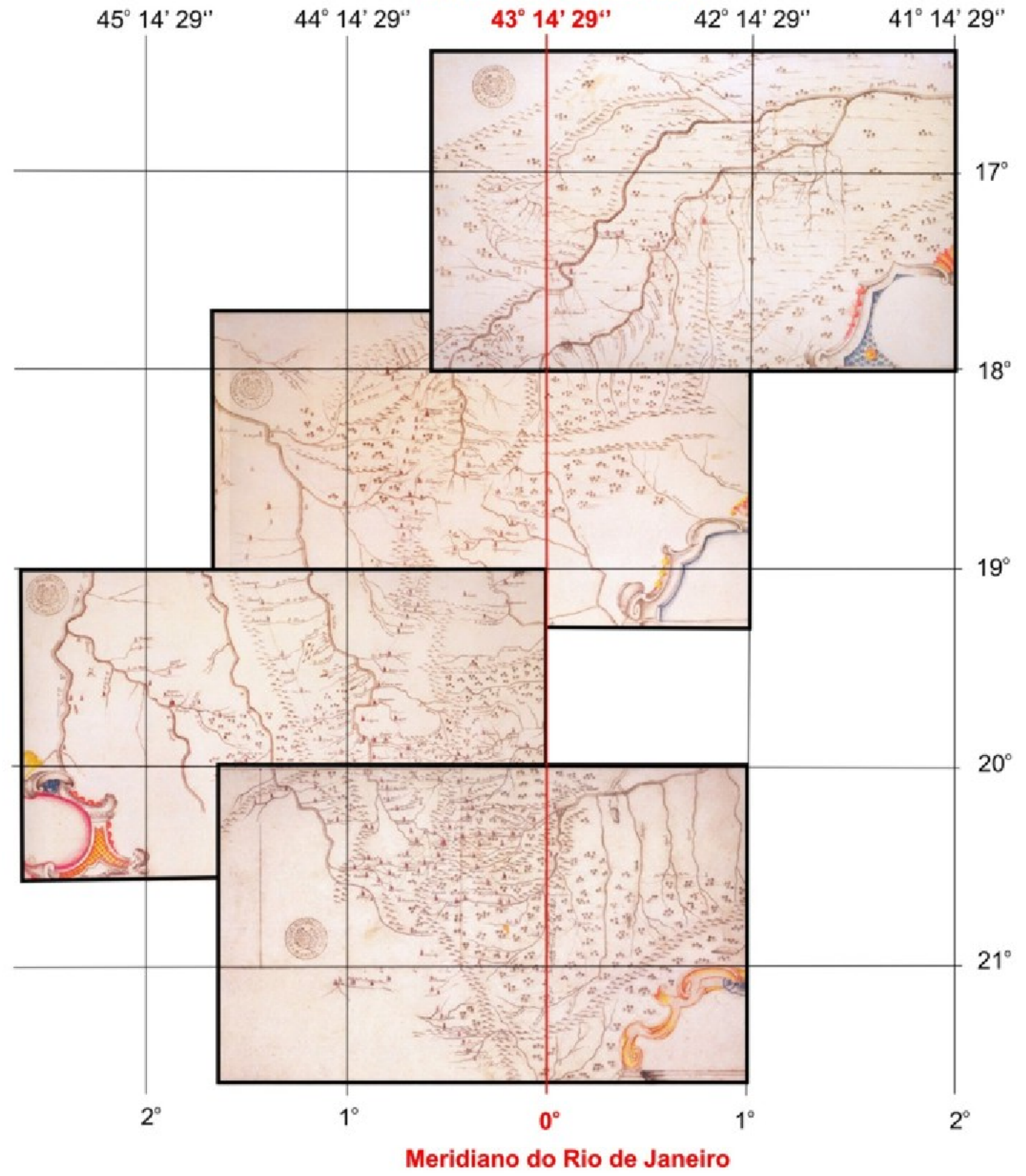

Fonte: Castro (2014) 
Figura 9 - Vetorização dos mapas dos padres matemáticos - 1734/35, representados por meio de mapa exaustivo e coleção de mapas destacando a hidrografia, as estradas, as vilas, as paróquias e as capelas
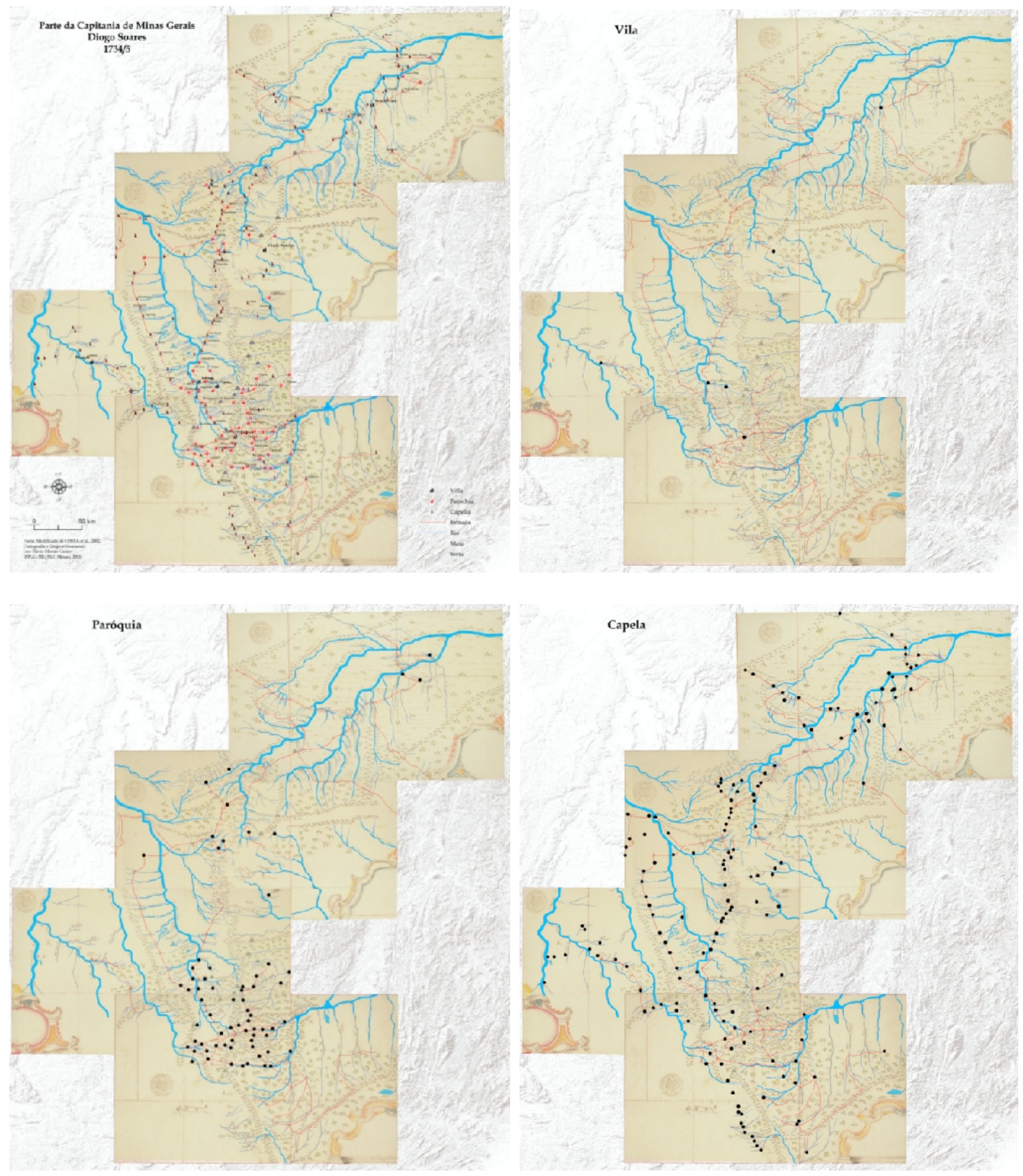

Fonte: modificado de Castro (2014) 
Figura 10 - Rede de meridianos e paralelos implícita nos mapas dos padres matemáticos de 1734/35 em relação ao atual; os pontos vermelhos representam 23 localidades (vilas e paróquias) selecionadas para a interpolação no MapAnalyst ${ }^{\circledR}$ (JENNY et al., 2007).

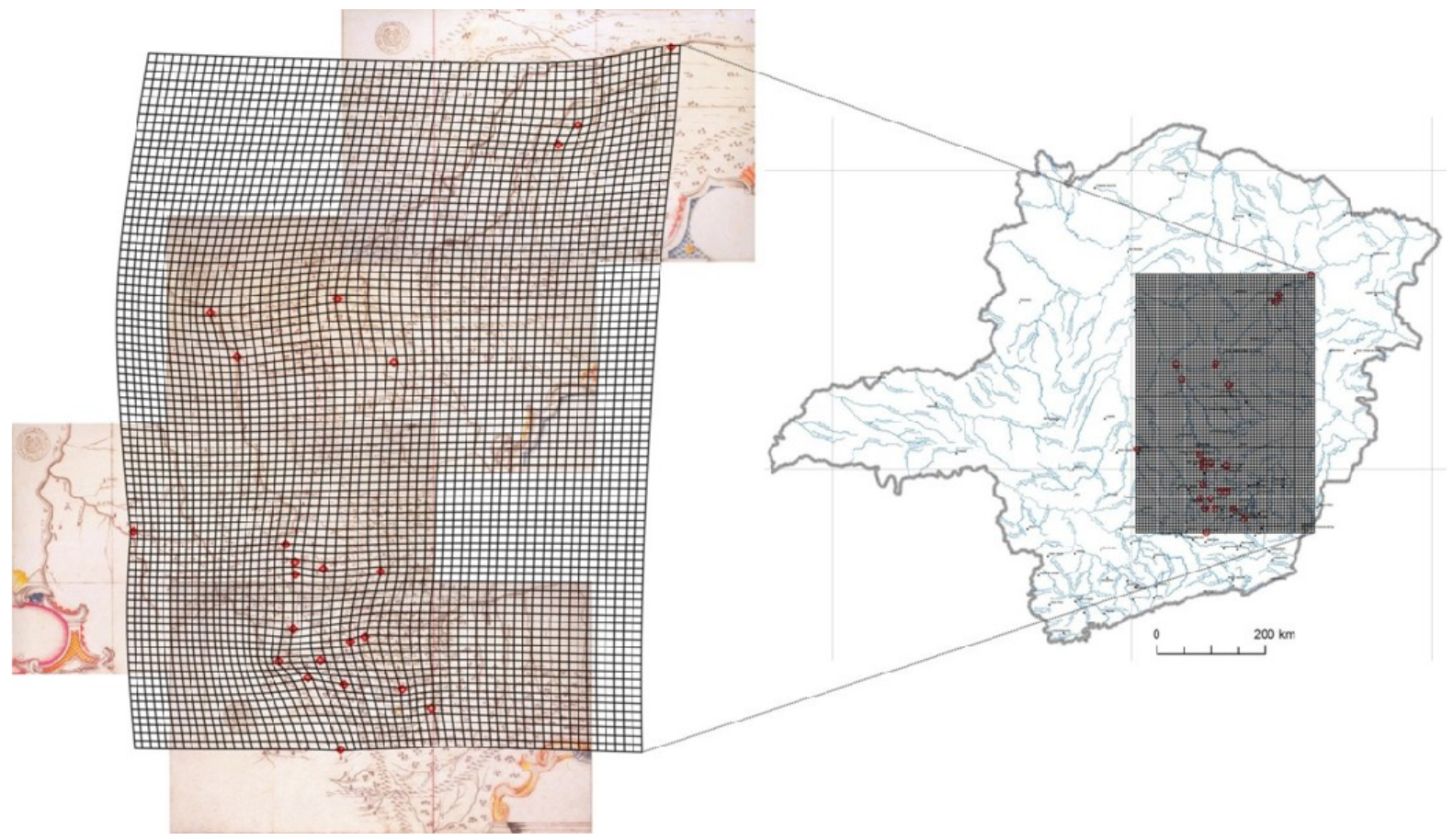

Figura 11 - Geovisualização dos mapas dos padres matemáticos - 1734/35 no Google Earth

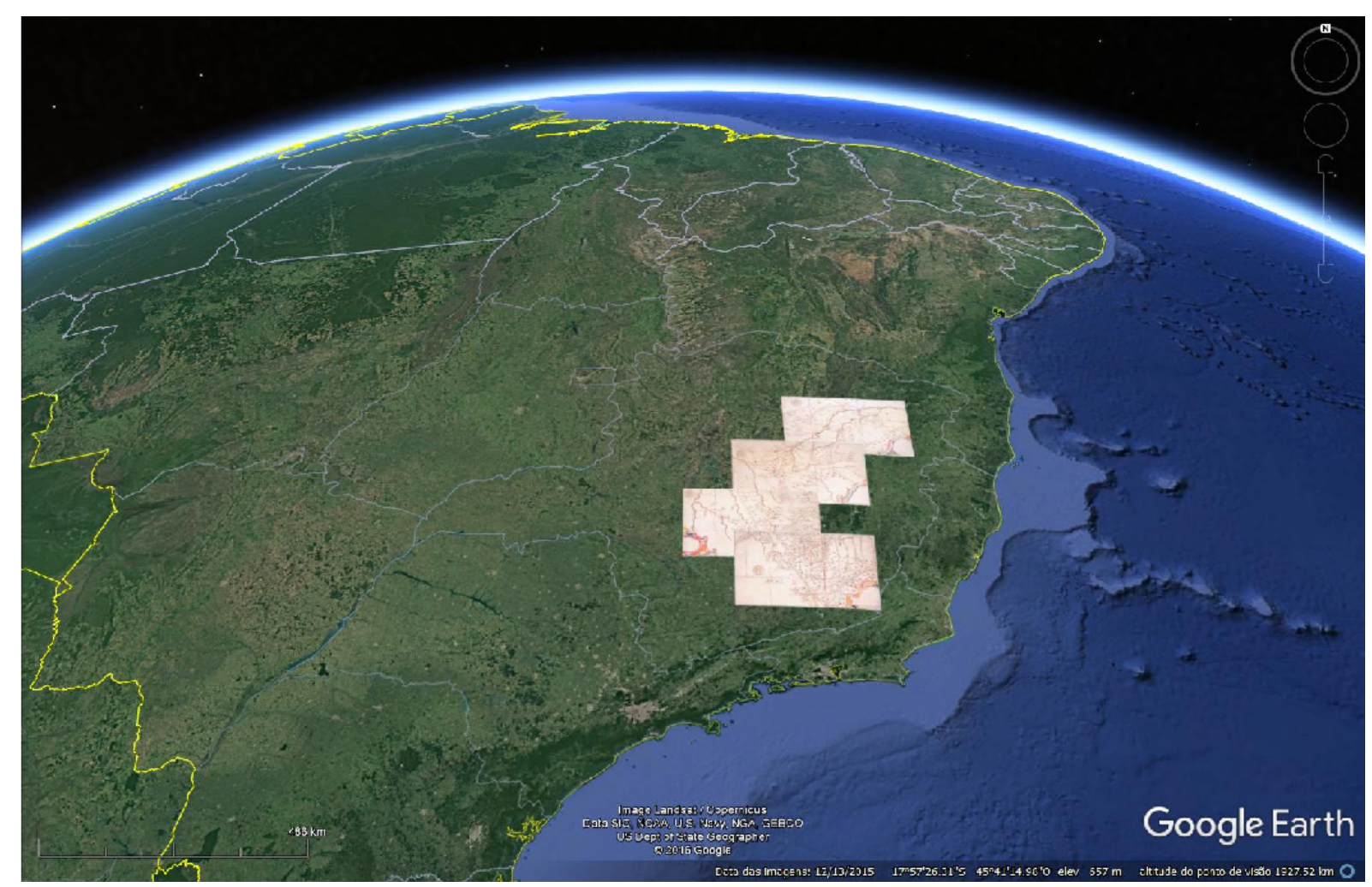

Fonte: elaborado pelo autor. 


\section{CONSIDERAÇÕES FINAIS}

A metodologia de georreferenciamento de mapas históricos mostrou-se eficiente no processo de recuperação de documentos pretéritos. Trata-se de um processo de georrefenciamento de dados espaciais e criação de banco de dados que permitem inúmeras manipulações e análises.

A geovisualização realizada foi simples, ou seja o referenciamento dos mapas históricos em imagem Google. Entretanto, algumas dificuldades foram identificadas, como por exemplo, no processo de exportação do ARC GIS ${ }^{\circledR}$ para o Google Earth Pro, as imagens são anexadas com baixa resolução, que comprometem a leitura do mapa.

As possibilidades de tratamento da informação espacial são amplas e diversificadas. Neste trabalho, foram apresentados brevemente a geovisualização em Google Earth Pro, dos mapas georreferenciados. Contudo, a técnica permite a sobreposição de dados vetoriais e sua manipulação em camadas, permite ainda que o usuário construa seus próprios mapas, entre outras opções de análise espacial.

As perspectivas estão voltadas para a interação das informações históricas georreferenciadas e vetorizadas com o usuário, especialmente, como recurso didático-pedagógico no ensino de História e Geografia.

\section{REFERÊNCIAS BIBLIOGRÁFICAS}

ALMEIDA, André Ferrand. A formação do espaço brasileiro e o projecto do Novo Atlas da América Portuguesa (1713-1748). Lisboa: Comissão Nacional para as Comemorações dos Descobrimentos Portugueses, 2001.

ANTONIL, André João. Cultura e opulência do Brasil por suas drogas e minas. Introdução e comentário crítico por Andrée Mansuy Diniz Silva. Lisboa: Comissão Nacional para as Comemorações dos Descobrimentos Portugueses, 2001.

BERTIN, J. Semiologie Graphique. Paris: Mouton-Gauthier-Villars, 1967.

BERTIN, Jacques. Ver ou ler: seleção de textos. São Paulo, n. 18, p. 45-62, 1988.

BOARD, C. A contribuição do geógrafo para a avaliação de mapas como meio de comunicação de informações. Geocartografia: Textos selecionados de cartografia teórica, Departamento de Geografia/FFLCH/USP, n. 3, 1994.

CARTWRIGHT, W.; PETERSON, M.; GARTNER, G. (Org.). Multimedia Cartography. Berlin: Springer-Verlag, 1999.

CASTRO, Dayan Magalhães. Visualização de dados geográficos urbanos na Web: estudo de caso na Região Metropolitana de Belo Horizonte. Dissertação de Mestrado, IGC/UFMG, Belo Horizonte, 2011.

CASTRO, J. F. M. Guaraciaba, 220 anos ou mais. Boletim Informativo Novos Tempos. Guaraciaba, 1998

CASTRO, J. F. M. et al.. Visualização Cartográfica dos Mapas de Minas Gerais dos Setecentos e Oitocentos: em destaque as bases urbanas. Anais do VI Seminário 
Latino-Americano de Qualidade de Vida Urbana e V Seminário Internacional de Estudos Urbanos. Belo Horizonte, PPGG-TIE/PUC Minas, 2006.

CASTRO, J. F. M. Comunicação cartográfica e visualização cartográfica. Boletim Paulista de Geografia, AGB, no. 87, p. 67-83, 2007.

CASTRO, J. F. M. Geografia Histórica e Cartografia de Minas Gerais no século XVIII. Relatório de Pesquisa de Pós-doutoramento. Porto: Faculdade de Letras da Universidade do Porto (FLUP), 2011.

CASTRO, J. F. M. História da Cartografia e Cartografia Sistemática. 1. ed. Belo Horizonte: Editora PUCMINAS, 2012.

CASTRO, José Flávio Morais. Cartografia histórica e geoprocessamento: o exemplo dos mapas dos padres matemáticos, de 1734, e do cálculo de rendimentos gerais do Bispado de Mariana, de 1752. Revista do Departamento de Geografia, USP, volume especial Cartogeo, 2014. p. 4-25.

CASTRO, J. F. M. Geoprocessamentomde mapas de Minas Gerais nos séculos XVIII-XIX. Belo Horizonte: Editora PUCMINAS, 2017.

CLARKE, K. C. Analytical and Computer Cartography. NJ: Prentice Hall, 1995. CORTESÃO, Jaime. História do Brasil nos velhos mapas. Lisboa: Imprensa Nacional/Casa da Moeda, 2009. v. 11, t. 1-2.

COSTA, Antônio Gilberto. et al. Cartografia de Minas Gerais: da capitania a província. Belo Horizonte: Editora UFMG; Lisboa: Kapa Editorial, 2002.

COSTA, Antônio Gilberto. (Org.). Cartografia da conquista do território das Minas. Belo Horizonte: Editora UFMG; Lisboa: Kapa Editorial, 2004.

CROMLEY, R. G. Digital Cartography. New Jersey: Prentice Hall, 1992.

DYKES, J., A. M. MacEachren, and M.-J. Kraak. (eds). Exploring geovisualization. Amsterdam: Elsevier. 2005.

GASPAR, J. A. Cartas e projeções cartográficas. Lisboa: Sociedade de Geografia de Lisboa, Lidel, 2000.

GASPAR, Joaquim Alves. Revisando a cartografia náutica portuguesa antiga do Atlântico: uma análise quantitativa. In: SIMPOSIO LUSO-BRASILEIRO DE CARTOGRAFIA HISTÓRICA, 3., Ouro Preto Anais... Ouro Preto, 2009. CD Rom.

JENNY, Bernhard; WEBER, Adrian; HURNI, Lorenz. Visualizing the planimetric accuracy of historical maps with MapAnalyst. Cartographica, v. 42, n. 1, p. 89-94, 2007.

JENNY, Bernhard; HURNI, Lorenz. Studying cartographic heritage: analysis and visualization of geometric distortions. Computers \& Graphics, v. 35, n. 2, p. 402411, 2011.

KOEMAN, C. O princípio da comunicação na cartografia. Geocartografia: Textos selecionados de cartografia teórica, Dep. de Geografia/FFLCH/USP, n. 5, 1995.

KOLACNY, A. Informação cartográfica: conceitos e termos fundamentais na cartografia moderna. Geocartografia: Textos selecionados de cartografia teórica, Departamento de Geografia/FFLCH/USP, n. 2, 1994. 
LE SANN, J. G. Documento cartográfico: considerações gerais. Revista Geografia e Ensino, Belo Horizonte, n. 1, v. 3. p. 3-17, 1983.

LIMA JÚNIOR, Augusto de. A capitania de Minas Gerais: origens e formação. 3. ed. Belo Horizonte: Edição do Instituto de História, Letras e Arte, 1965.

LONGLEY, P. A.; GOODCHILD, M. F.; MAGUIRE, D. J.; DHIND, D. W. Sistemas e ciência da informação geográfica. Porto Alegre: Bookman, 2013.

MACEACHREN, A. M. How Maps Work: representation, visualization and design. New York: Guilford Press, 1995.

MACEACHREN, A. M. and KRAAK, M. J. Research challenges in geovisualization. Cartography and Geographic Information Science. 28(1): 3-12. 2001.

MAGUIRE, D. J.; GOODCHILD, M. F.; RHIND, D. W. (Eds.) Geographical

Information Systems: Principles and Aplications. New York: Wiley, 1991.

MARTINELLI, M. Curso de Cartografia Temática. São Paulo: Contexto, 1991.

OLIVEIRA, L. Estudo metodológico e cognitivo do mapa. Tese de Livre-Docência, IG/USP, São Paulo, 1978.

PETCHENIK, B. B. Cognição em Cartografia. Geocartografia: Textos

selecionados de cartografia teórica, Departamento de Geografia/FFLCH/USP, n. 6, 1995.

PETERSON, M. P. Interactive and Animated Cartography. New Jersey: PrinticeHall, 1995.

RAMOS, C. S. Visualização cartográfica e cartografia multimídia: Conceitos e tecnologia. São Paulo: Editora da UNESP, 2005.

ROCHA, José Joaquim da. Geografia histórica da Capitania de Minas Gerais. Descrição geográfica, topográfica, histórica e política da Capitania de Minas Gerais. Memória histórica da Capitania de Minas Gerais. Estudo crítico: Maria Efigênia Lage de Resende; transcrição e colação de textos: Maria Efigênia Lage de Resende e Rita de Cássia Marques. Belo Horizonte: Fundação João Pinheiro, Centro de Estudos Históricos e Culturais, 1995. (Coleção Mineiriana).

SCHMIDT, M.A.R.; DELAZARI, L.S.; NADAL, M.A.D. Geovisualização e Sistemas de Informação Geográfica. Anais do II Simpósio Brasileiro de Geomática e V Colóquio Brasileiro de Ciências Geodésicas, 2007.

SIMIELLI, M. E. R. O mapa como meio de comunicação: implicações no ensino da geografia do $1^{\circ}$ grau. Tese de Doutoramento, FFLCH/USP, São Paulo, 1986.

SIMÕES, Carlos Enrique Hernández. Geovisualização analítica: desenvolvimento de um protótipo de um sistema analítico de informações para a gestão da coleta seletiva de resíduos urbanos recicláveis. Dissertação de Mestrado. POLI/USP, São Paulo, 2010.

\section{Agradecimento}

Um agradecimento especial à Profa. Dra. Ana Márcia Moreira Alvim (coordenadora do PPGG-TIE/PUCMINAS) pela leitura cuidadosa, pelas críticas e sugestões. 